\title{
Modelagem Paramétrica aplicada a projeto de produto de Tecnologia Assistiva
}

\author{
Parametric Modeling applied to an Assistive Technology Product Design \\ - Adriana Bolaños-Mora \\ PGDesign, UFRGS, Brasil \\ adrianabmora@gmail.com \\ - Karen Mello Colpes \\ PGDesign, UFRGS, Brasil \\ karencolpes@gmail.com \\ - Tânia Luisa Koltermann da Silva \\ PGDesign, UFRGS, Brasil \\ tania.koltermann@ufrgs.br \\ - Régio Pierre da Silva \\ PGDesign, UFRGS, Brasil \\ regio@ufrgs.br
}

\begin{abstract}
The article presents an Assistive Technology product design, which aims to overcome the medical product idea, considering its aesthetic configuration, and aims to change the concept of mass production into custom manufacturing. The methodological process was to obtain the user's body 3D model, for subsequent parameterization by using the Grasshopper's Rhinoceros Plugin software. It is believed that the digital manufacturing process provides a more flexible production, especially in the prototyping and testing stages, which seems a competitive advantage over companies that do not use them.
\end{abstract}

Keywords: Modelagem Paramétrica, Tecnologia Assistiva, Grasshopper, Customização em Massa

\section{Introdução}

A tecnologia nos surpreende dia a dia, mostrando como viver pode ser cada vez mais fácil e prazeroso. Constantemente fazemos uso de artefatos que nos auxiliam em diversas tarefas, potencializam os nossos sentidos e simplificam coisas que antes eram complexas, tornando real o que outrora era quase impossível.

As técnicas de manufatura têm evoluído significativamente, conseguindo aliar o melhor da produção sob encomenda às vantagens da fabricação em larga escala originárias da revolução industrial. Neste contexto, para Tedeschi (2014), a Modelagem Paramétrica éapresentada como um novo método de produção baseado em modelos digitais, destacando-se pela capacidade de se alterar rapidamente dados variáveis predefinidos, conhecidos como parâmetros.

Segundo Florio (2011), o uso de parâmetros para definir a geometria de elementos construtivos, tem se mostrado cada vez mais eficaz no processo de projeto, possibilitando a obtenção de geometrias complexas e introduzindo a possibilidade de criar e manipular novas famílias de formas e de superfícies curvas.

Neste contexto, uma modelagem desse tipo exige que as formas geradas sejam agrupadas em componentes constituídos por esses parâmetros, de modo a facilitar a manipulação de acordo com a necessidade do usuário. Para

isso, existem alguns plug-ins, como o Grasshopper, com uma interface gráfica intuitiva que têm facilitado o trabalho de designers e arquitetos que não possuem conhecimentos sólidos em programação (Florio, 2011).

Desta forma, o processo torna-se otimizado visando evitar o retrabalho e conferir maior celeridade às fases de projeto $\mathrm{e}$ confecção do produto. Tal propriedade não favorece apenas o processo de projeto de produto, mas, também viabiliza a adaptação destes às características de seu usuário final, permitindo certa "personalização" do produto, a chamada Customização em Massa (Celani \& Pupo, 2008).

Entretanto, ainda se encontram campos de produção que por costume, economia, falta de conhecimento ou todos juntos, continuam a usar métodos de fabricação em massa, o que tende a dificultar a adaptação do produto gerado às particularidades do corpo de cada indivíduo. Principalmente no que tange à produção de artigos que favoreçam a qualidade de vida e capacidade de autonomia das pessoas, caso dos produtos de Tecnologia Assistiva (TA). Para estes, a Customização de Massa tem se apresentado como a solução para uma maior aceitação e satisfação do usuário e melhor eficácia do produto. Pois a Customização em Massa aumenta a rentabilidade através de uma sinergia em se elevar o valor percebido pelo cliente e a redução dos custos de produção e logística (Jianxin, Qinhai \& Mitchell, 2003).

Sabe-se que no Brasil a Tecnologia Assistiva é considerada uma área de conhecimento de característica interdisciplinar, conglomerando produtos, recursos, metodologias, estratégias, 
práticas e serviços que objetivam promover a funcionalidade relacionada à atividade e participação de pessoas com deficiência, alguma incapacidade ou mobilidade reduzida, visando proporcionar-lhes maior autonomia, melhoria na qualidade de vida, e sua inclusão social (Brasil, 2009). Desta forma, entende-sequea TA vaialém daspessoas com deficiência, abrangendo a todos que em algum momento de suas vidas seja por incapacidade temporária ou idade avançada - precise de alguma tecnologia que assista a sua carência.

$\mathrm{Na}$ procura por favorecer o processo de fabricação de produtos de TA, almejando uma produção de menor custo, a indústria optou pela padronização de processos, dimensões, densidades, e até mesmo uma "estandardização imposta" do própriocorpohumano. Noentanto, tal procedimentoacarretou certa inflexibilidade e rigidez que tornaram estes produtos difíceis de se adequar às particularidades e preferências de cada pessoa. Aliado a isto tem-se as dificuldades de se realizar procedimentos de medição antropométrica em pessoas com deformação física ou movimentos involuntários, assim, para estas pessoas, algumas variáveis, tornam-se impossíveis de serem obtidas (Brendler, 2013).

Diante do exposto, é notório que as medidas antropométricas são de suma importância para o desenvolvimento de produtos que estejam cada vez mais adequados às características e particularidades físicas de cada usuário. Aqui radica a vantagem do uso da modelagem paramétrica, tendo na digitalização tridimensional uma importante ferramenta, para a obtenção das variáveis antropométricas, às quais algumas deficiências afetam significativamente. Servindo ainda como forma de customização ou personalização destes produtos, factível através da alteração de determinados parâmetros.

$\mathrm{Na}$ esfera da TA, os projetos de produto deveriam permitir maior personalização das variações antropométricas para atender as singularidades de cada pessoa com deficiência, quanto mais suas preferências estéticas. No entanto, conforme dito anteriormente, a realidade do mercado de produtos de TA é a massificação, o que leva a produtos limitados do ponto de vista de adequação ao corpo do usuário.

Este estudo, que se originou a partir de uma atividade didática da disciplina de "Processos de Fabricação Digital como Ferramenta de Projeto" do Programa de Pós-Graduação em Design e Tecnologia da UFRGS (PGDesign), pretende ressaltar não apenas as vantagens da Modelagem Paramétrica a serviço do desenvolvimento de produtos de TA, mas também destacar o potencial existente na customização destes produtos, principalmente para pessoas que apresentem alguma deformação física. Uma vez que a essência da customização em massa reside na capacidade de se desenvolver produtos e serviços de acordo com a diversidade de perfil e necessidades de cada indivíduo. (Jianxin, \& Mitchell, 2004). A fim de exemplificar esta potencialidade, optou-se por aplicar tais metodologias ao projeto de um Colar Cervical personalizado, objeto importante para reabilitação de pessoas com algum tipo de deficiência temporária ou permanente que dificulte a sustentação do pescoço, de utilização aparente e que possui uma estética pouco atrativa.

O colar cervical é um produto facilmente encontrado no mercado atual que poderia ser pensado como um elemento adicional para compor um visual, um acessório funcional, porém, os modelos disponíveis possuem pouca possibilidade de adaptação ao corpo, além de uma estética robusta (Basso, 2012). Desta forma, utilizou-se da adaptabilidade e versatilidade proporcionadas pela Modelagem Paramétrica (Anderson, 2010), para projetar o Colar Cervical Customizado (CCC).

\section{Procedimento Metodológico}

Para a obtenção da digitalização tridimensional (modelo 3D) do sujeito, foi utilizado o dispositivo Kinect da Microsoft, pois, além de ser um dispositivo portátil e de fácil manuseio, possui baixo custo em relação aos scanners convencionais de digitalização tridimensional disponíveis no mercado (Brendler, 2013).

Mas, para consegui-la foram necessárias várias tentativas (Figura 1), algumas não foram passíveis de utilização, pois suas características não permitiam a geração da malha e, portanto, da programação no Grasshopper (Khabazi, 2010). Ocorre que este sistema de digitalização 3D é muito sensível e pela falta de experiência, alguns ruídos foram criados na imagem por fios de cabelos soltos, rugas de tecido na roupa e movimentos do usuário. Além do fator de o topo da cabeça não ter sido digitalizado, ficando a malha incompleta.

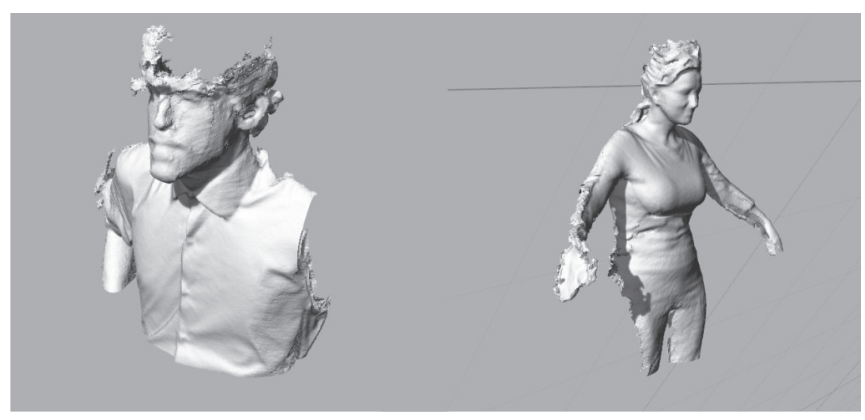

Figura 1: Tentativas de obtenção do modelo 3D do usuário.

Após estes testes, tornou-se mais fácil entender a maneira correta de digitalizar o usuário e aplicá-la, figura 2. A terceira digitalização partiu de uma área um pouco abaixo dos ombros até a coroa da cabeça, com os cabelos totalmente presos e assegurando-se de que a malha ficasse completamente preenchida, ou seja, fechada. 


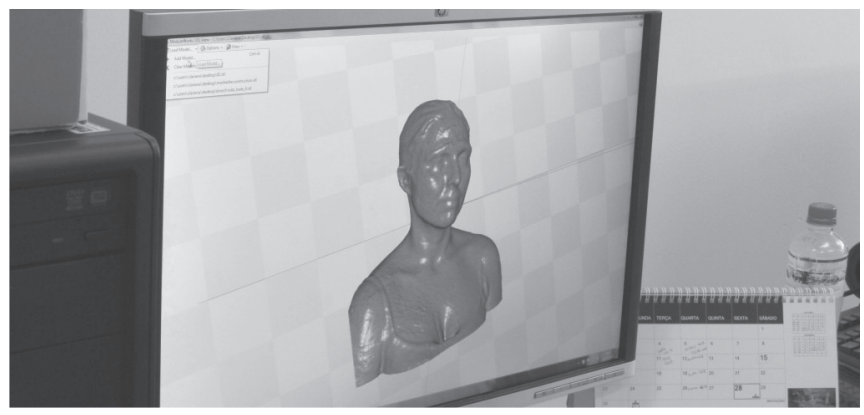

Figura 2: Terceira tentativa de digitalização.

As imagens digitalizadas foram convertidas em uma malha tridimensional, permitindo a sua edição e processamento a fim de gerar o modelo 3D, conforme Figura 3.

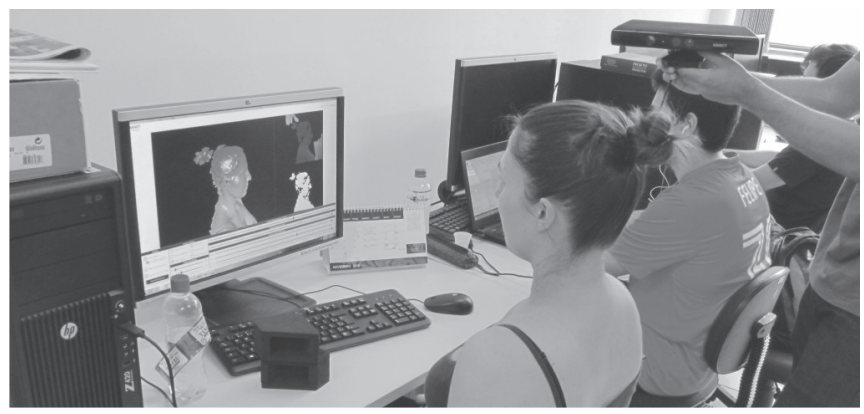

Figura 3: Modelo 3D do usuário.

O modelo gerado foi exportado em formato STL (Stereolitography) para que pudesse ser interpretado e trabalhado no software de modelagem 3D, Rhinoceros $₫$, com a utilização do plugin Grasshopper.

\section{Parametrização 3D do colar cervical}

A partir do modelo escaneado e de um template com o formato da projeção frontal do colar, a região do pescoço foi seccionada em vários planos, gerando uma série de curvas que estruturaram a modelagem paramétrica das superfícies do colar cervical (figura 4).

As curvas foram, então, separadas para a geração das duas metades do colete (sobrepostas nos encaixes) (figura 5).

Em seguida, foram feitos recortes nas curvas para a compatibilização final com o template (figura 6) e geração das superfícies internas e externas do colar (figura 7).

Por fim, algoritmos de subdivisão regular das superfícies internas e externas geraram padrões hexagonais para as peças que compuseram as duas metades do colar (Figura 8).

\section{Resultado}

O resultado obtido centrou-se em expor a versatilidade da Modelagem Paramétrica aplicada a um Projeto de Produto

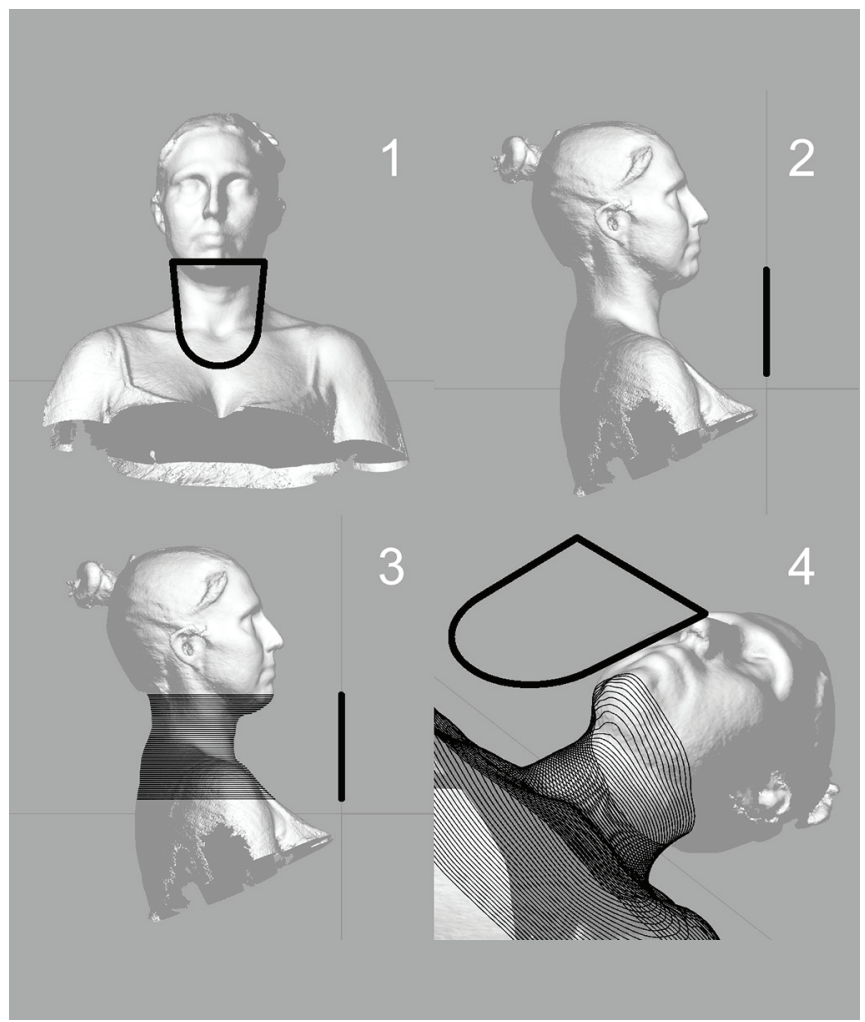

Figura 4: Vistas do template sobre o modelo (1 e 2), vista com as curvas geradas (3) e perspectiva (4).

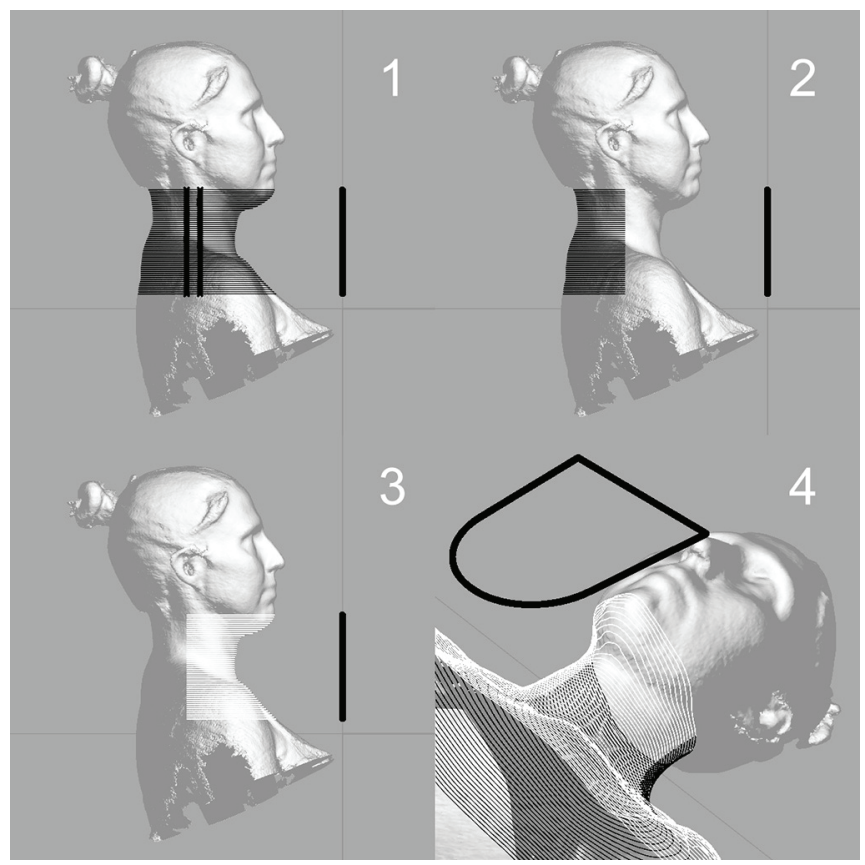

Figura 5: Vista da separação das curvas (1), vistas com as curvas separadas por cor (parte posterior em preto e frontal em branco) (2 e 3) e perspectiva. 


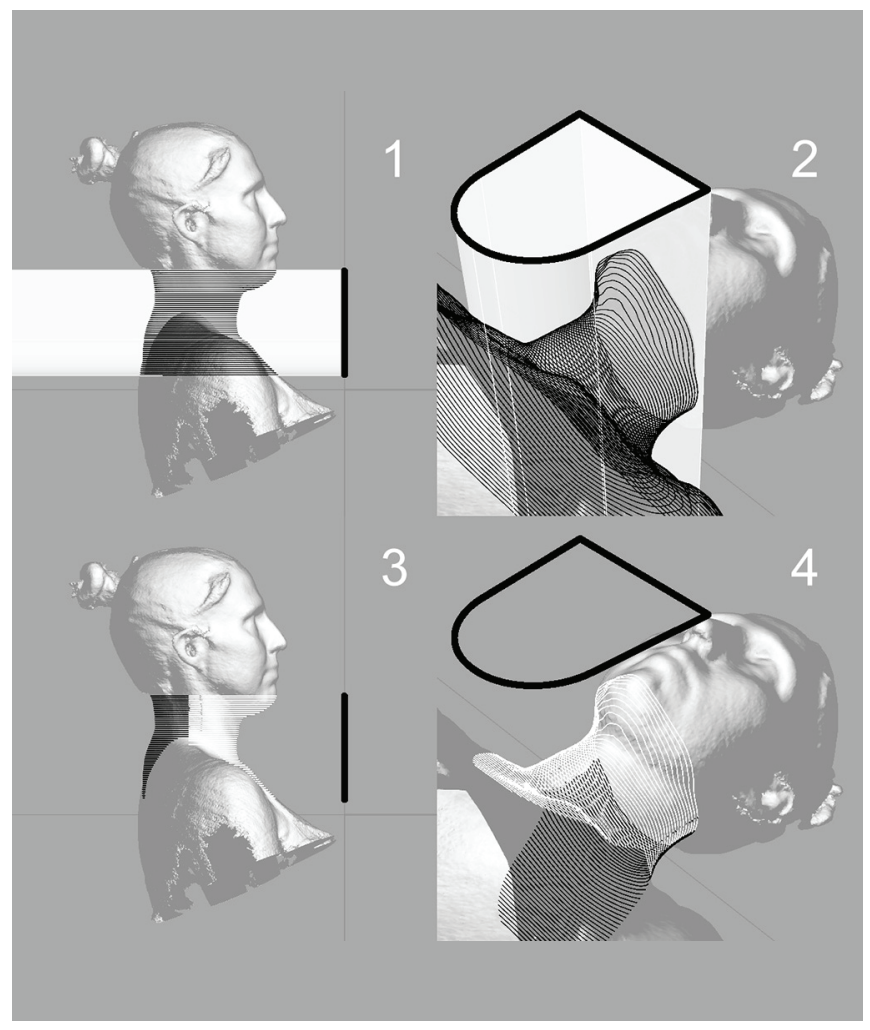

Figura 6: Operações de intersecção para o recorte das curvas e compatibilização final com o template.

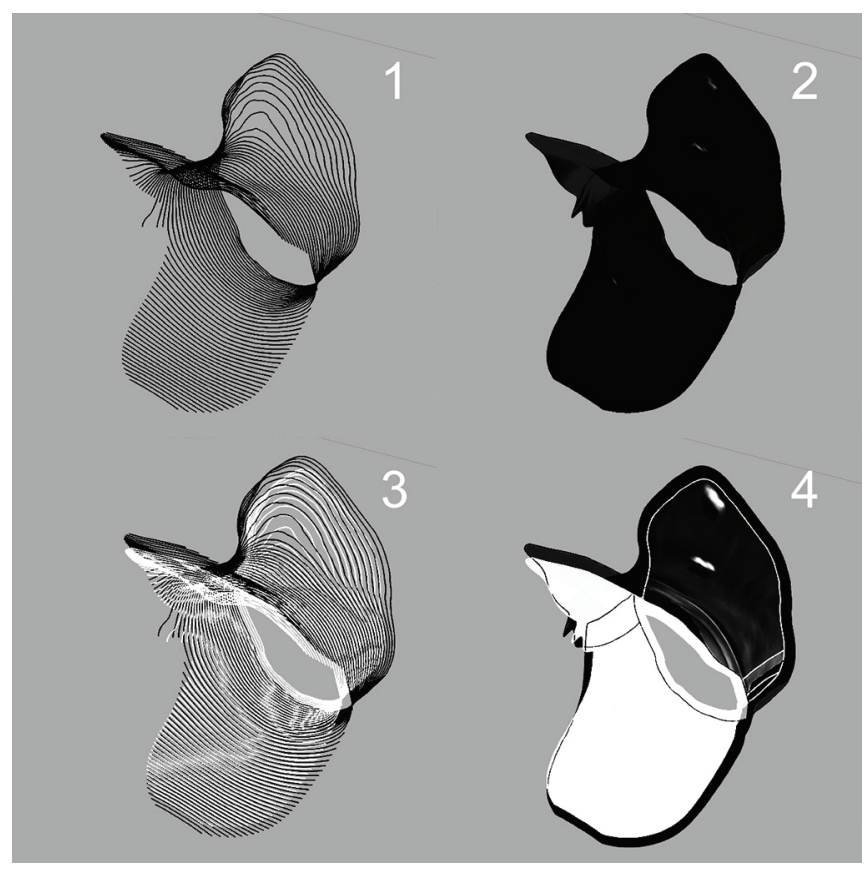

Figura 7: Modelagem, a partir das curvas, das superfícies internas e externas de ambas as peças do colar.

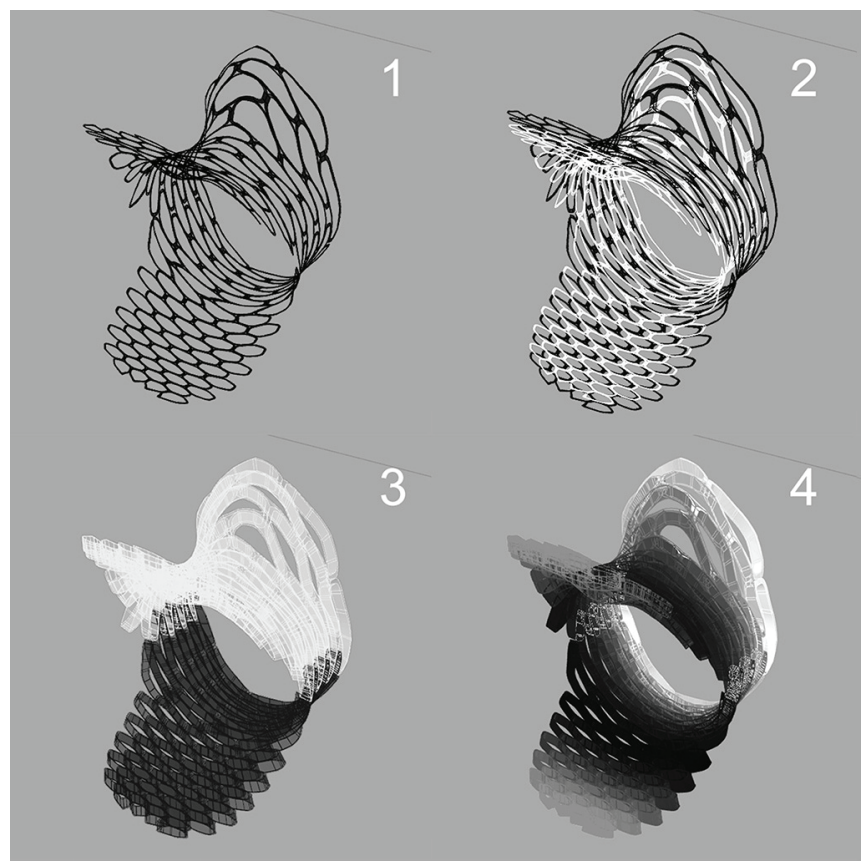

Figura 8: Subdivisão das superfícies (1, 2 e 3) e modelo do colar finalizado (4).

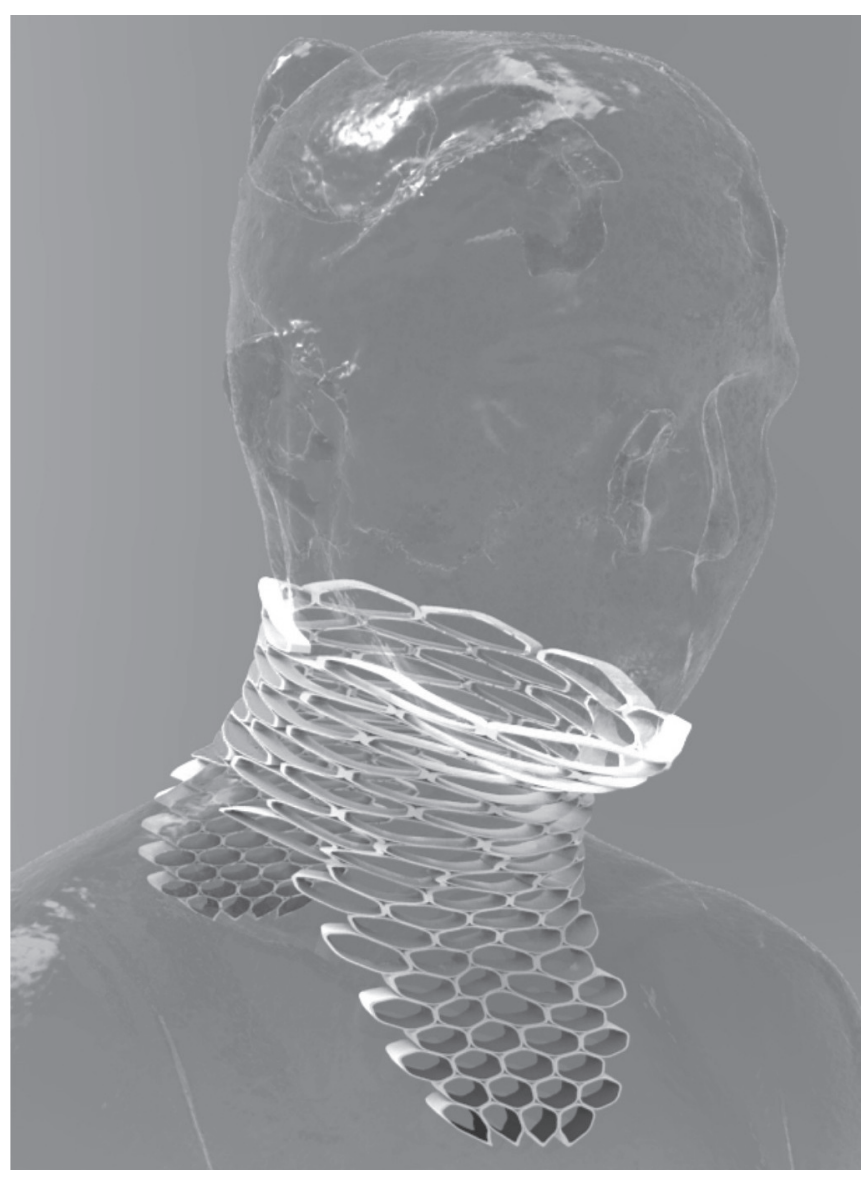

Figura 9: Colar Cervical Customizado. 
de TA, o Colar Cervical Customizado (Figura 9).

O desenvolvimento deste produto, no software Rhinoceros ${ }^{\circledR}$ com ajuda do plugin Grasshopper oferece ao designer a capacidade de gerar modelos paramétricos de formas complexas através de uma interface gráfica intuitiva e de adaptá-los facilmente a novos e diferentes padrões antropométricos por meio da alteração de alguns parâmetros.

Consequentemente, favoreceu o projeto do produto customizado em questão, ideal para pessoas com algum tipo de deficiência temporária ou permanente que dificulte a sustentação do pescoço. Fica evidente que esta tecnologia viabiliza, torna mais atrativa do ponto de vista estético, ágil, econômica e menos trabalhosa a fabricação de produtos feitos sob medida ou customizados, ratificando assim, o valor da modelagem paramétrica.

Porém, é importante salientar que esta proposta ainda se encontra em desenvolvimento e fase de testes. Assim, novos ensaios serão necessários para averiguar questões como material, conforto e segurança, em prol da qualidade de vida dos usuários.

\section{Discussão}

A Modelagem Paramétrica aplicada ao projeto de Produtos de TA pode simbolizar uma revolução mercadológica para os usuários desta tecnologia, devido a uma mudança de perspectiva pela adoção de modelos que amparam este desenvolvimento. Assim, os produtos de TA que eram projetados com base em modelos médicos, passam a adotar um modelo mais dinâmico, atento às especificidades de seu usuário, ampliando seu potencial de uso e melhorando a qualidade de vida destas pessoas.

Isto porque quando a TA é percebida como um sinalizador da deficiência, esta pode se tornar uma barreira para que o indivíduo participe de atividades diárias (Oishi, Mitchell \& Van Der Loos, 2010),pois, este percebe sua deficiência ou limitação amplificada pela utilização da tecnologia em questão, gerando constrangimento ao usuário, fato que se vê refletido no elevado índice de abandono destes recursos assistivos (Benedetto, 2011).

Diante do exposto, percebe-se uma oportunidade para pesquisadores, indústria e comércio conhecerem estes usuários, possibilitando projetar um mundo mais adequado para eles, a partir de suas reais necessidades (Clarksom \& Coleman, 2015), tanto em nível individual quanto em nível coletivo (Benedetto, 2011).

Percebe-se ainda que projetos de produtos de TA como um todo podem ser desenvolvidos através da Modelagem Paramétrica, pois o procedimento proporciona a flexibilidade necessária de adequação ao corpo dos diferentes biótipos de usuário e a possibilidade de se obter uma estética atraente e inovadora (Jianxin, \& Mitchell, 2004).

\section{Agradecimentos}

Gostaríamos de agradecer à Professora Msc. Clariana Fischer Brendler e ao bolsista Leonardo Kratz Mendes, do Laboratório Virtual Design - VID do PGDesign da UFRGS, por terem colaborado neste trabalho com a digitalização tridimensional para a obtenção do modelo 3D.

\section{Referências}

Anderson, C. (2010). In the next industrial revolution, atoms are the new bits. Wired Magazine. Disponível em: $\quad$ http://www.wired.com/magazine/2010/01/ff_ newrevolution/

Basso L. (2012). A Contribuição do Designer no Projeto de Recursos de Tecnologia Assistiva: Proposta de Intervenção Colaborativa. Dissertação de Mestrado apresentada ao Programa de Pós-graduação em Design - UFRGS, Brasil. Benedetto, I. (2011). Contribuições Metodológicas para o Desenvolvimento de Produtos em Tecnologia Assistiva. Dissertação de Mestrado apresentada ao Programa de Pós-graduação em Design - UFRGS, Brasil.

Bersch, R. (2013). Introdução à Tecnologia Assistiva. Assistiva - Tecnologia e Educação. Porto Alegre, RS.

Brasil. (2009). Subsecretaria Nacional de Promoção dos Direitos da Pessoa com Deficiência. Comitê de Ajudas Técnicas. Tecnologia Assistiva - CORDE. Brasília.

Brendler, C. F. (2013). Método para Levantamento de Parâmetros antropométricos utilizando um digitalizador 3D de baixo custo. Dissertação de Mestrado apresentada ao Programa de Pós-graduação em Design - UFRGS, Brasil. Celani, G. \& Pupo, R. (2008). Prototipagem Rápida e Fabricação Digital para Arquitetura e Construção: Definições e Estado da Arte no Brasil. Cadernos de Pósgraduação em Arquitetura e Urbanismo.

Florio, W. (2011). Modelagem Paramétrica, Criatividade e Projeto: Duas Experiências com Estudantes de Arquitetura, Gestão e Tecnologia de Projetos, Volume 6, número 2, ISSN: 19811543, DOI: 10.4237/gtp.v6i2.211.

Jianxin J., Qinhai M., \& Mitchell M. T. (2003). Towards high value-added products and services: mass customization and beyond. Technovation, 23, 809-821. Disponível em: www.elsevier.com/locate/technovation

Jianxin J., \& Mitchell M. T.(2004). Customizability analysis in design for mass customization. ComputerAided Design, 36 745-757. Disponível em: www.elsevier. com/locate/cad

Khabazi, Z. (2010). Algoritmi Generativi con Grasshopper. Disponível em: ttp://issuu.com/pabloherrera/docs/ algoritmigenerativi?e $=155$ 0707/2677764

Oishi, M. M. K., Mitchell, I. M. \& Van Der Loos, H. F. M. (2010). Design and use of assistive technology: Social, technical, ethical, and economic challenges.

Tedeschi, A. (2011). Parametric Architecture with Grasshopper. Primer. 1Ed.

Tedeschi, A. (2014). AAD_Algorithms-Aided Design. Parametric Strategies Using Grasshopper ${ }^{\circledR}$ English Edition. Edizioni Le Penseur. 